\title{
Association between a $15 q 25$ gene variant, nicotine-related habits, lung cancer and COPD among 56307 individuals from the HUNT study in Norway
}

\begin{abstract}
Maiken E Gabrielsen ${ }^{1}$, Pål Romundstad ${ }^{2}$, Arnulf Langhammer ${ }^{2}$, Hans E Krokan ${ }^{1}$ and Frank Skorpen ${ }^{\star 3}$
Genetic studies have shown an association between single-nucleotide polymorphisms on chromosome 15q25 and smokingrelated traits and diseases, such as quantity of smoking, lung cancer and chronic obstructive pulmonary disease (COPD). A discussion has centred on the variants and their effects being directly disease related or indirect via nicotine addiction. To address these discrepancies, we genotyped the single-nucleotide polymorphism rs16969968 in the CHRNA5/A3/B4 gene cluster at chromosome 15q25, in 56307 individuals from a large homogenous population-based cohort, the North Trøndelag Health Study (HUNT) in Norway. The variant was examined in relation to four different outcomes: lung cancer, loss of lung function equivalent to that of COPD, smoking behaviour and the use of smokeless tobacco (snus). Novel associations were found between rs 16969968 and the motivational factor for starting to use snus, and the quantity of snus used. Our results also confirm and extend previous findings for associations between rs 16969968 and lung cancer, loss of lung function equivalent to that of COPD, and smoking quantity. Our data suggest a role for rs 16969968 in nicotine addiction, and the novel association with snus strengthens this observation.
\end{abstract}

European Journal of Human Genetics (2013) 21, 1293-1299; doi:10.1038/ejhg.2013.26; published online 27 February 2013

Keywords: genetic association; lung cancer; nicotine addiction; COPD; snus

\section{INTRODUCTION}

Tobacco-related deaths reached 100 million individuals during the 20th century. It is estimated to reach 1 billion deaths during the 21 st century and each year 5.4 million deaths world wide can be attributed to cigarette smoking. ${ }^{1}$ In Norway, a steady decline in daily smoking has been observed since the mid-1990s and today $17 \%$ of the adult population smoke on a daily or occasional basis (http://www.ssb.no/royk/).

Lung cancer and chronic obstructive pulmonary disease (COPD) are both strongly associated with tobacco smoking. ${ }^{2}$ Lung cancer is the leading cause of cancer death world wide with $\sim 1.1$ million deaths per year, ${ }^{1}$ while COPD is the 4 th leading cause of death, ${ }^{3}$ killing 2.75 million people world wide in $2002{ }^{4}$ A gene region on chromosome 15q25, containing the nicotine-acetylcholine receptor (nAChRs) subunits CHRNA5/A3/B4, has been found to be associated with lung cancer in several genome-wide association studies (GWAS) $)^{5-9}$ and replication studies. ${ }^{10-12}$ GWAS have also shown association with COPD at the same loci. ${ }^{13}$ A number of large studies also report an association of this region with smoking-related traits and nicotine addiction. ${ }^{14-24}$ Associations with several SNPs and distinct loci within the CHRNA5/A3/B4 region have been reported in these studies. ${ }^{15,16,25}$

The CHRNA5/A3/B4 genes encode subunits of nAChRs. These are ligand-gated ion channels classified into two main categories, neuronal and muscular. They are activated both by the endogenous neurotransmitter acetylcholine and chemicals such as nicotine and its metabolites, including nicotine specific nitrosamines. The receptors, believed to have a role in nicotine dependence, lead to nicotinemediated increase of dopamine in the nucleus accumbens (reviewed in Improgo et $a^{26}$ ). nAChRs have also been found to be expressed in lung tissue where subsequent activation may promote cell proliferation and inhibition of apoptosis. ${ }^{27-29}$ Current evidence points to plausible biological associations of $\mathrm{nAChR}$ with both nicotine dependence and lung cancer.

In addition to cigarettes, a different nicotine-containing tobacco product, snus (often referred to as Swedish snus), is available in Norway. This is a moist smokeless tobacco product typically placed under the upper lip and kept there (without chewing). ${ }^{30}$ The use of snus in Norway has steadily increased, especially amongst the younger population. Data from Statistics Norway (http://www.ssb.no/royk/) show that around $8 \%$ of the adult population use snus on a daily or occasional basis. Among the youngest age group (16-24 years), as many as $25 \%$ of males use snus daily. Although snus contains many of the same harmful substances as cigarettes, it is considered less harmful, but is believed to be similar in producing nicotine dependence. ${ }^{30}$

In this study, we report a novel association between the rs16969968 polymorphism in CHRNA5 and the use of snus. We detect two distinct associations related to the use of snus; one with the quantity of snus used per month and a second to whether the reason for starting to use snus was related to an effort to reduce or quit smoking. We also replicate previously reported associations with the

${ }^{1}$ Department of Cancer Research and Molecular Medicine, Faculty of Medicine, Norwegian University of Science and Technology, Trondheim, Norway; ${ }^{2}$ Department of Public Health and General Practice, Faculty of Medicine, Norwegian University of Science and Technology, Trondheim, Norway; ${ }^{3}$ Department of Laboratory Medicine, Children's and Women's Health, Faculty of Medicine, Norwegian University of Science and Technology, Trondheim, Norway

${ }^{*}$ Correspondence: Professor F Skorpen, Department of Laboratory Medicine, Children's and Women's Health, Faculty of Medicine, NTNU, P0-Box 8905, Trondheim N-7491, Norway. Tel: +47 725733 32; Fax: +47 725764 00; E-mail: frank.skorpen@ntnu.no

Received 18 September 2012; revised 22 January 2013; accepted 24 January 2013; published online 27 February 2013 
CHRNA5/A3/B4 gene cluster by examining the rs16969968 polymorphism in relation to lung cancer risk, smoking quantity and loss of lung function equivalent to that of COPD in a large homogenous population cohort (the HUNT cohort of the North Trøndelag County, Norway).

\section{MATERIALS AND METHODS}

\section{Populations studied}

The Nord-Trøndelag Health Study (HUNT) is a comprehensive populationbased study having collected data of the entire adult population aged 20 years or above in three consecutive surveys, HUNT 1 (1984-86), HUNT 2 $(1995-97)^{31}$ and HUNT 3 (2006-08). ${ }^{32}$ The studies comprise data from questionnaires, interviews and clinical examinations. All participants in HUNT $2(n=65237)$ and HUNT $3(n=50807)$ provided blood samples. DNA has been prepared from peripheral blood leucocytes from all participants in HUNT 2 and is stored in the HUNT biobank (Levanger, Norway). Approximately 36000 individuals participated both in the HUNT 2 and HUNT 3 studies (Figure 1). ${ }^{31,32}$

The Lung Study in HUNT invited random samples of participants in HUNT 2 $(5 \%, n=2791)$ and HUNT $3(10 \%, n=5068)$. In addition, participants in the two studies reporting having had asthma, COPD or asthma-related symptoms were invited, totalling 8150 from HUNT 2 and 7391 from HUNT 3. All participants were subjected to lung function measurements (spirometry), measurement of bone mineral density and went through an interview. ${ }^{33}$

\section{Study characteristics}

Lung cancer. Lung cancer diagnosis was available from the Cancer Registry of Norway. Data in the Cancer Registry of Norway are based on morphological diagnosis from all pathology departments in Norway and a written report from the clinical departments. ${ }^{34}$ Cases were identified by linking the HUNT database to the Cancer Registry of Norway via the unique national personal identity number. Only individuals who developed lung cancer after participation in the HUNT 2 study (1995) (Figure 1) and who were diagnosed with lung cancer as the primary tumour were included in the analysis. Only de-identified data were available for researchers.

Loss of lung function. The loss of lung function was based on spirometric data from the HUNT 3 lung study (Figure 1). Individuals with loss of lung function equivalent to moderate or severe COPD were identified based on the following standard criteria: prebronchodilator $\mathrm{FEV}_{1} / \mathrm{FVC}<0.7$ and $\mathrm{FEV} 1 \%$ predicted $<80$ and/or having received the diagnosis COPD from their medical doctor. Controls were individuals with lung function $\mathrm{FEV}_{1} / \mathrm{FVC}>0.7$ and $\mathrm{FEV} 1 \%$ predicted $>80$. In the present study, reference equations developed from the same region was used. ${ }^{35}$

Smoking. Smoking status was categorised into never, former and current smoker based on answers to the HUNT 2 main questionnaire. Never smokers reported 'I have never smoked daily' and had not reported any other smokingrelated information. Former smokers reported having previously smoked and/ or years since smoking cessation, whereas current smokers reported smoking daily and/or reported a number of cigarettes smoked daily. The variable eversmoker was computed combining current and former smokers. Individuals were also asked to report the number of cigarettes smoked per day or used to smoke per day if quitted smoking. Smoking burden in pack-years was calculated by smoking duration multiplied with daily number of cigarettes divided by 20 .

Snus. The use of snus was categorised into never, former and current users according to answers to the HUNT 3 main questionnaire. Questions on this subject were not included in HUNT 2. Never snus users reported 'No, I have never used snus'. Former snus users reported having previously used snus, while current snus users reported using snus on a daily or occasional basis. Individuals reporting their age when starting to use snus, snus consumption per month or a motivational factor for starting to use snus were also classified as current snus user. Individuals were also asked to report the number of boxes of snus consumed per month and this variable was used in the snus consumption analysis. Participants were asked 'When you started to use snus, was this in order to quit or reduce smoking?' Individuals who answered 'yes in order to reduce smoking' and 'yes in order to quit smoking' were merged to create the binary variable 'Motivation for starting to use snus.'

Genotyping. The SNP, rs16969968, from the CHRNA5/A3/B4 gene cluster on 15 q25 was genotyped. Genotyping was performed at the HUNT biobank using TaqMan genotyping assay (Applied Biosystems, Foster City, CA, USA) and performed on an Applied Biosystems 7900HT Fast real-Time PCR System using $10 \mathrm{ng}$ of genomic DNA. Each 384-well plate contained four negative and four positive controls. Four samples were used as quality controls for genotype consistency and were included on every plate (384 wells) genotyped and the call rate cutoff was set to $90 \%$. The genotyping success rate was $98.6 \%$ and quality score for each individual genotype was $>90$ (mean 99.7). The minor a

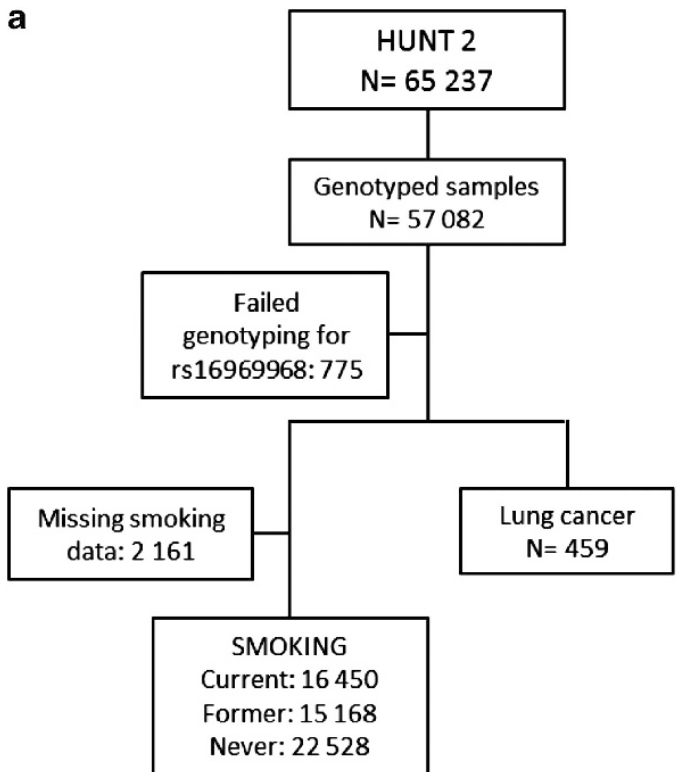

b

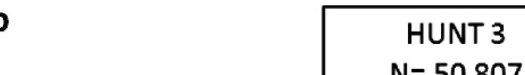

$\mathrm{N}=50807$

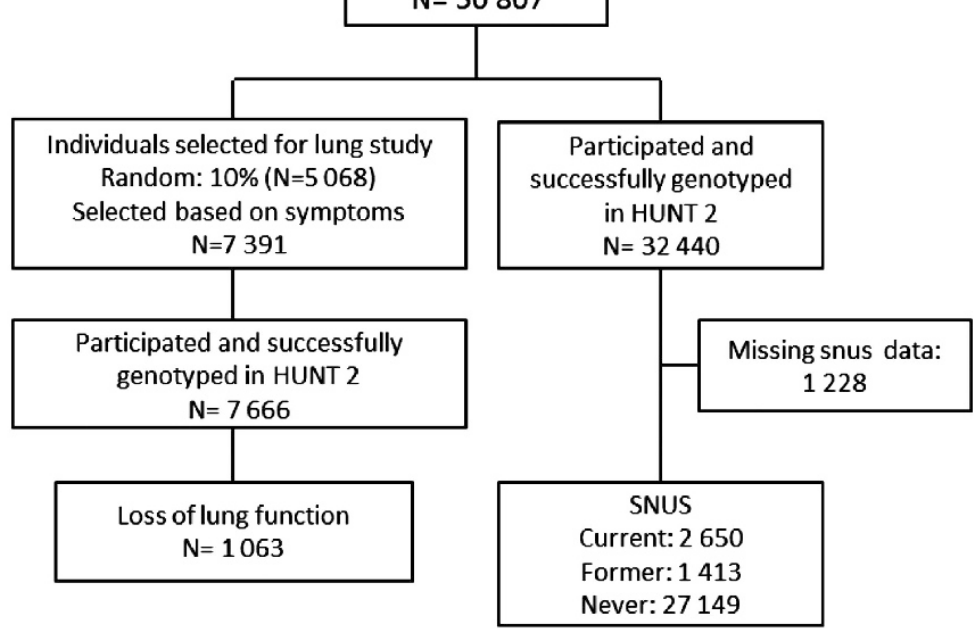

Figure 1 Flow-chart visualising the number of individuals for the different traits/diseases selected from the HUNT 2 (a) and HUNT 3 (b) studies. 
allele frequency was in agreement with HapMap data $(\mathrm{MAF}=0.385$ and 0.337 , respectively) and the genotype frequencies conformed to Hardy-Weinberg equilibrium (test for Hardy-Weinberg equilibrium $P=0.2$ ). Genotyping was performed for all individuals with available DNA (57082) and laboratory personnel were blinded to any phenotypic status.

\section{Statistical analysis}

All analyses were performed in PASW Statistics 18 (SPSS Inc. Released 2009. PASW Statistics for Windows, Version 18.0; Chicago, IL, USA). Binary outcomes were analysed using logistic regression, continuous outcomes using linear regression and Cox regression was used to estimate hazard ratios (HR) for lung cancer.

Both a genotype specific (GG vs GA and AA) and per allele (additive) model was calculated and adjusted for age, sex and cigarettes per day (CPD). Additional analyses were performed, stratified on smoking status and sex, and a $P$-value for trend was calculated for the per allele model. For the Cox-regression analysis the end of follow-up (EOF) date was the 31 December 2009. 'Person-time' was calculated by subtracting the date of participation in HUNT 2, or date of diagnosis, for controls and cases, respectively, from the EOF date and dividing the number of days by 365.25 . Heterogeneity between groups was tested by adding an interaction term in a separate regression analysis to test the interaction between the SNP and the trait. Differences in genotype distributions between the different groups of smokers and snus users were tested using a $\chi^{2}$-test. A two-sided $P$-value $<0.05$ was considered statistically significant.

\section{Ethics}

This study has been approved by the Regional Committees for Medical and Health Research Ethics. A written consent was signed by all participants in the HUNT study. The study conforms to the declaration of Helsinki.

\section{RESULTS}

Table 1 gives an overall overview of the HUNT 2 and HUNT 3 cohorts included in the present study. Numbers of individuals are given according to genotype, smoking and snus status, lung cancer and loss of lung function.

\section{Lung cancer and loss of lung function}

A statistical significant association was found between rs16969968 and the risk of lung cancer in the Cox regression with a HR of 1.45 (95\% CI: $1.25-1.67, P=4.60 \times 10^{-7}$ ) per allele (A) adjusted for age, sex and CPD (Table 2). Sex was not a significant variable in the regression analysis (Table 2 ) and when analyses were run stratified by sex (adjusted for age and CPD), no statistical significant heterogeneity was observed between sexes $(P=0.096$, data not shown $)$. When stratified by smoking status, statistical significant association with lung cancer was seen only in current smokers ( $\mathrm{HR}=1.51,95 \% \mathrm{CI}$ : 1.29-1.77, $P=2.95 \times 10^{-7}$ ), while a minor non-significant effect was observed in former smokers $(\mathrm{HR}=1.25,95 \% \mathrm{CI}$ : $0.97-1.62$, $P=0.088$ ) and no association was observed in never smokers $(P=0.382)$. Heterogeneity was observed between the groups (P-het $0.036)$.

A statistical significant association was found between the variant allele (A) and the loss of lung function equivalent to COPD ( $\mathrm{OR}=1.36,95 \% \mathrm{CI}: 1.19-1.55, P=4.25 \times 10^{-6}$ ) (Table 2). Sex was a significant variable in the regression analysis (Table 2). However, when analyses were run stratified by sex, the interaction term was not significant $(P=0.137)$ (data not shown). A significant association was found in current and former smokers $(\mathrm{OR}=1.48,95 \% \mathrm{CI}$ : $1.25-1.76, \quad P=4.84 \times 10^{-6}$ and $\mathrm{OR}=1.25,95 \%$ CI: $1.06-1.48$, $P=0.007$, respectively), whereas no association was seen in never smokers $(P=0.88)$. Heterogeneity was observed between the smoking status groups (P-het 0.011).
Table 1 Characteristics of the HUNT 2 and HUNT 3 population, overall and per genotype.

\begin{tabular}{lcccc}
\hline & & \multicolumn{4}{c}{ Genotype } \\
& Overall & $G G$ & $G A$ & $A A$ \\
\hline HUNT 2 & & & \\
$\quad$ Total (n) & 56307 & 24800 & 25035 & 6472 \\
Males (\%) & $26839(47.7)$ & $11868(44.2)$ & $11914(44.4)$ & $3057(11.4)$ \\
Female (\%) & $29468(52.3)$ & $12932(43.9)$ & $13121(44.5)$ & $3405(11.6)$ \\
Mean age (SD) & $50.0(17.1)$ & $50.0(17.1)$ & $49.9(17.1)$ & $49.6(17.0)$ \\
Smokers & & & & \\
$\quad$ Never (\%) & $22528(40.0)$ & $10032(44.5)$ & $9933(44.1)$ & $2563(11.4)$ \\
Former (\%) & $15168(26.9)$ & $6848(45.2)$ & $6665(43.9)$ & $1655(10.9)$ \\
Current (\%) & $16450(29.2)$ & $6919(42.1)$ & $7491(45.5)$ & $2040(12.4)$ \\
Lung cancer & & & & \\
Cases (\%) & 459 & $155(33.8)$ & $227(49.4)$ & $77(16.8)$ \\
Controls (\%) & 55823 & $24634(44.1)$ & $24798(44.4)$ & $6391(11.5)$ \\
& & & & \\
HUNT 3 & & & & \\
Total (n) & 32440 & 14384 & 14372 & 3684 \\
Males (\%) & $14775(45.5)$ & $6548(44.3)$ & $6551(44.3)$ & $1676(11.3)$ \\
Females (\%) & $17665(54.5)$ & $7836(44.4)$ & $7821(44.3)$ & $2008(11.4)$ \\
Mean age (SD) & $58.2(13.4)$ & $58.4(13.4)$ & $58.2(13.3)$ & $57.7(13.3)$ \\
Snus users & & & & \\
$\quad$ Never (\%) & $27149(83.7)$ & $12024(44.3)$ & $12045(44.4)$ & $3080(11.3)$ \\
Former (\%) & $1413(4.4)$ & $623(44.1)$ & $634(44.9)$ & $156(11.0)$ \\
Current (\%) & $2650(8.2)$ & $1195(45.1)$ & $1134(42.8)$ & $321(12.1)$
\end{tabular}

\begin{tabular}{|c|c|c|c|c|}
\hline \multicolumn{5}{|c|}{ HUNT 3 lung study } \\
\hline \multicolumn{5}{|c|}{ Loss of lung functiona } \\
\hline Cases (\%) & 1063 & 412 (38.8) & 499 (46.9) & $152(14.3)$ \\
\hline Controls (\%) & 5301 & $2420(45.6)$ & $2289(43.2)$ & $592(11.2)$ \\
\hline
\end{tabular}

Abbreviation: HUNT, the North Trøndelag Health Study.

Questions on the use of snus and spirometry data were available from the HUNT 3 study only. The $\%$ of smoking and snus status are calculated from their total population $(n)$, respectively. Missing data for smoking and use of snus were $3.8 \%$.

andividuals with loss of lung function participated in the HUNT 3 lung study (Figure 1).

\section{Smoking and snus}

Individuals homozygous for the variant A allele, when compared with non-carriers, smoked on average 1.11 cigarettes more per day $\left(P\right.$-trend $\left.=3.15 \times 10^{-25}\right)$ (Table 3$)$, had smoked on average 0.83 years longer $\left(P\right.$-trend $\left.=1.11 \times 10^{-6}\right)$ and had smoked on average 1.81 pack-years more $\left(P\right.$-trend $\left.=3.01 \times 10^{-23}\right)$ when adjusted for age and sex. No difference in genotype frequencies was observed between never smokers and ever smokers (Pearson's $\chi^{2}$-test, $2 \mathrm{df}$, $P=0.07)$. However, a statistical significant difference in the distribution of genotypes was seen between current and former smokers (Table 1, Pearson's $\chi^{2}$-test, $2 \mathrm{df}, P<10^{-4}$ ).

A significant association was found between the variant A allele and monthly snus consumption. Individuals homozygous for the A allele used on average 0.53 boxes of snus more per month compared with individuals not carrying the A allele $\left(P\right.$-trend $\left.=4.29 \times 10^{-3}\right)$ (Table 3). If only individuals who reported to be never smokers were considered, the difference between the two homozygous groups was 1.25 boxes per month $(\mathrm{BPM})\left(P\right.$-trend $\left.=2.0 \times 10^{-3}\right)$. Carriers of the A allele were also more likely to have started to use snus in order to reduce or quit smoking $(P=0.001)$ (Table 4$)$. No difference in the genotype frequencies between never snus users and ever snus users was detected (Pearson's $\chi^{2}$-test, $2 \mathrm{df}, P=0.719$ ). 
Table 2 HR for lung cancer and OR for loss of lung function equivalent to COPD according to rs16969968 allele distribution.

\begin{tabular}{|c|c|c|c|c|c|c|c|}
\hline \multirow[b]{2}{*}{ Lung cancer } & \multirow[b]{2}{*}{ Case subjects } & \multirow[b]{2}{*}{ Control subjects } & \multirow[b]{2}{*}{ HR unadjusted ${ }^{a}$} & \multicolumn{4}{|c|}{$95 \% \mathrm{Cl}$ for $\mathrm{HR}$} \\
\hline & & & & $H R^{\mathrm{b}}$ & Lower & Upper & P-value \\
\hline rs16969968 per allele & $383^{d}$ & $28369^{d}$ & 1.48 & 1.45 & 1.25 & 1.67 & $4.60 \times 10^{-7}$ \\
\hline rs16969968 GG & $125^{d}$ & $12386^{d}$ & Ref & Ref & - & - & $3.30 \times 10^{-6}$ \\
\hline rs16969968 GA & $189^{d}$ & $12685^{d}$ & 1.51 & 1.47 & 1.17 & 1.84 & $8.85 \times 10^{-4}$ \\
\hline rs16969968 AA & $69^{d}$ & $3298^{d}$ & 2.16 & 2.08 & 1.55 & 2.79 & $1.07 \times 10^{-6}$ \\
\hline Sex & & & & 0.94 & 0.76 & 1.17 & 0.592 \\
\hline Age & & & & 1.06 & 1.05 & 1.06 & $1.67 \times 10^{-51}$ \\
\hline CPD & & & & 1.03 & 1.02 & 1.05 & $5.87 \times 10^{-9}$ \\
\hline
\end{tabular}

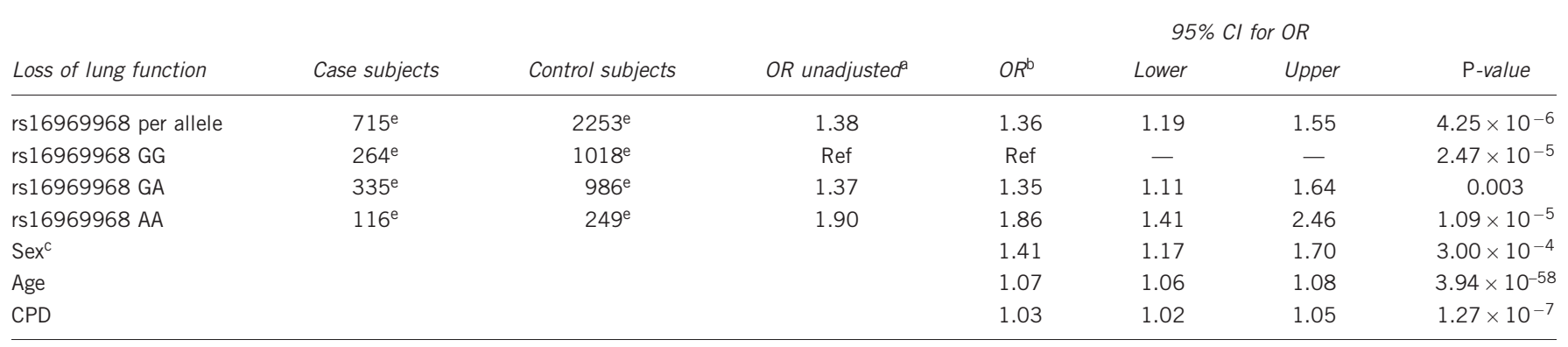

Abbreviations: $\mathrm{Cl}$, confidence of interval; COPD, chronic obstructive pulmonary disease; CPD, cigarettes per day; HR, hazard ratio; OR, odds ratio.

Complete Cox-regression analysis model for lung cancer and logistic regression analysis for loss of lung function.

Sex, age and CPD are covariates in the regression analysis. The $P$-value shows whether these variables are significant in the model and the HR and OR, respectively, show their contribution to disease risk.

${ }^{a}$ Adjusted for sex and age only.

bHR and OR are adjusted for age, sex and CPD.

${ }^{\mathrm{C}}$ Reference sex is female.

dOnly individuals with valid data for smoking quantity (CPD) were included in the analysis.

eOnly individuals with valid person-time and valid data for smoking quantity (CPD) were included in the analysis.

\section{DISCUSSION}

In this study, we demonstrate novel associations between rs16969968 and the reason for starting to use snus being related to smoking reduction or cessation, and to the quantity of snus used. Our results also confirm and extend previous findings of association between the rs16969968 A allele with increased risk of lung cancer, loss of lung function equivalent to COPD and with increased tobacco consumption. ${ }^{10,12,13}$ Previous studies have speculated whether the lung cancer association is confounded by COPD. ${ }^{36}$ Owing to a limited number of lung cancer cases participating in the HUNT Lung Study, we had insufficient power to detect potential confounding by COPD. We performed several statistical tests throughout the present study. With increasing number of tests, the chance of false-positive findings increases, which means that the results should be interpreted with caution. However, the main analyses were based on a clear hypothesis, and the different tests were related (not independent). Thus, formal adjustments for the number of tests, such as the Bonferroni method, would probably be too conservative.

Smoking is the major contributor to the risk of lung cancer. Lips et $a l^{10}$ argue that the $1.2 \mathrm{CPD}$ increase found for homozygous carriers of the A allele cannot account for the increased risk of lung cancer conveyed by rs16969968. However, an increase in the number of years smoked or pack-years could increase the lung cancer risk substantially more. ${ }^{37}$ In the present study, individuals homozygous for the A allele had an average of 1.8 pack-years more and had smoked 0.83 years longer than non-carriers, which may contribute substantially to the lung cancer risk. Even so, association with the SNP seems to be related to the quantity, and duration of smoking or use of snus as opposed to the status of use (never or ever user). This is also emphasised by the fact that a larger proportion of current smokers are homozygous for the risk allele (AA) than in the former smoker group.
Previous research has shown that the consumption of snus is associated with an increased probability of being a former smoker. ${ }^{38}$ The novel association between the A allele of rs16969968 and the motivation for starting to use snus being related to smoking reduction or cessation can be seen as a proxy for nicotine dependence, as it is likely that individuals with a stronger nicotine dependence substitute cigarettes with smokeless tobacco in order to reduce or quit smoking. A Swedish study from 2003 showed that $30 \%$ of former smokers in Sweden used snus while quitting smoking. ${ }^{30}$ This fits well with one of the important hallmarks of nicotine addiction, namely the tendency to relapse to tobacco use. ${ }^{39}$ There was no difference in the mean number of CPD between the 'Yes' and 'No' groups regarding the motivational factor for starting to use snus. Adding $\mathrm{CPD}$ as a covariate in the analysis did not change the regression coefficient, however, because of a reduction in the number of individuals in the analysis, the $P$-value was not significant (data not shown). As for smoking quantity, individuals homozygous for the risk allele used more snus per month (0.53 BPM more). However, when considering snus users who reported to be never smokers, this difference was substantially larger (1.25 BPM). These findings strengthen the evidence for an association between rs16969968 and nicotine dependence.

rs16969968 is a non-synonymous SNP, introducing a substitution of aspartic acid (D) with asparagine (N) at amino-acid position 398 (D398N) of the CHRNA5 protein. It is a likely candidate to mediate a functional effect, although other SNP variants and haplotypes ${ }^{20,40-42}$ in the $15 \mathrm{q} 25$ region might modulate the effect on lung cancer and nicotine dependence. ${ }^{15,16,25}$

Research by Bierut et ll $^{15}$ shows that the variant A allele of rs16969968 leads to reduced receptor activity and that individuals carrying the A allele may require larger amounts of nicotine to achieve 
Table 3 Association of rs 16969968 with smoking quantity in CPD and snus quantity in BPM

\begin{tabular}{|c|c|c|c|c|c|}
\hline Smoking quantity & $n^{\mathrm{a}}$ & \multicolumn{4}{|c|}{$95 \% \mathrm{Cl}$ mean $\mathrm{CPD}$} \\
\hline \multicolumn{6}{|l|}{ Genotype } \\
\hline $\mathrm{GA}$ & 12882 & 11.66 & 11.55 & 11.77 & \\
\hline AA & 3370 & 12.13 & 11.92 & 12.35 & \\
\hline Abs diff between homozygous & & 1.11 & & & $3.15 \times 10^{-25}$ \\
\hline \multicolumn{6}{|l|}{ Men } \\
\hline $\mathrm{GG}$ & 6468 & 12.60 & 12.42 & 12.78 & \\
\hline GA & 6504 & 13.29 & 13.11 & 13.47 & \\
\hline$A A$ & 1698 & 13.78 & 13.43 & 14.14 & \\
\hline Abs diff between homozygous & & 1.18 & & & $8.11 \times 10^{-12}$ \\
\hline$A A$ & 1672 & 10.42 & 10.18 & 10.67 & \\
\hline Abs difference between homozygous & & 1.04 & & & $2.79 \times 10^{-17}$ \\
\hline
\end{tabular}

\begin{tabular}{|c|c|c|c|c|c|}
\hline \multirow[b]{2}{*}{ Snus quantity } & \multirow[b]{2}{*}{$n^{\mathrm{b}}$} & \multirow[b]{2}{*}{ Mean BPM } & \multicolumn{2}{|c|}{$95 \% \mathrm{Cl}$ mean $B P M$} & \multirow[b]{2}{*}{ P-trend } \\
\hline & & & Lower & Upper & \\
\hline GG & 1652 & 5.33 & 5.10 & 5.57 & \\
\hline $\mathrm{GA}$ & 1597 & 5.86 & 5.62 & 6.10 & \\
\hline $\mathrm{AA}$ & 436 & 5.86 & 5.40 & 6.30 & \\
\hline \multicolumn{6}{|l|}{ By sex } \\
\hline Men & 3323 & 5.85 & 5.65 & 6.06 & $6.00 \times 10^{-3 c}$ \\
\hline Women & 362 & 4.15 & 3.57 & 4.74 & $0.436^{c}$ \\
\hline
\end{tabular}

Abbreviations: BPM, boxes per month; $\mathrm{Cl}$, confidence interval; CPD, cigarettes per day.

Multiple linear regression model for CPD and snus used in boxes per month, means are adjusted for age and sex.

aOnly individuals with valid data for smoking quantity (CPD) were included.

bonly individuals with valid data for snus quantity used per month were included.

cP-trend for the increase in snus consumption (BPM) seen.

Table 4 Motivation for start using snus related to smoking, reduction/cessation; "Yes"/"No"

\begin{tabular}{|c|c|c|c|c|c|c|}
\hline Genotype & n, “No.” (\%) & n, "Yes" (\%) & \multicolumn{4}{|c|}{$95 \% \mathrm{Cl}$ for $\mathrm{OR}$} \\
\hline Per allele (A) & 2383 & 1560 & 1.17 & 1.06 & 1.29 & 0.001 \\
\hline GA & $1050(44.1)$ & $702(45.0)$ & 1.09 & 0.95 & 1.26 & 0.218 \\
\hline AA & $250(10.5)$ & 217 (13.9) & 1.46 & 1.18 & 1.81 & $4.55 \times 10^{-4}$ \\
\hline
\end{tabular}

Abbreviations: $\mathrm{Cl}$, confidence interval; OR, odds ratio.

Logistic regression analysis for the association between rs 16969968 and the motivation behind starting to use snus was performed adjusted for age and sex. Only individuals reporting a

motivational factor for starting to use snus were included in the analysis. The variable was created in the following manner: participants were asked 'When you started to use snus, was this in

order to quit or reduce smoking?' Individuals who answered 'yes in order to reduce smoking' and 'yes in order to quit smoking' were merged to create the binary variable 'Motivation for starting to use snus.'

the same level of dopamine release. This is in concordance with our finding that individuals carrying the A allele tend to smoke more (1.1 CPD more for AA homozygotes) and also continue to smoke for longer period of time ( 0.83 years for AA homozygotes). This increase in smoking load is likely to greatly increase the risk of lung cancer. Thorgeirsson and Stefansson ${ }^{37}$ argue that, based on the Doll-Peto equation, a 5\% increase in smoking duration (eg. from 20-21 years) would bring about an $\sim 30 \%$ increase in lung cancer risk, strengthening the possible role of the polymorphism in nicotine addiction and smoking behaviour, but does not exclude an independent risk on lung cancer in never smokers.

Based on the findings in this and related studies together with the knowledge of the function of nAChRs, it is plausible that the SNP rs16969968 has an effect on smoking behaviour linked to nicotine dependence. The increased risk of the A allele with lung cancer seems in our study to be restricted to current and perhaps former smokers. Even though this is a large population-based study, the number of lung cancer patients, especially among never smokers, is low and gives 
limited power to detect association in never smokers as they constitute a minority of the lung cancer patients. However, several larger studies fail to detect an association in never smokers ${ }^{12,43,11}$ and collectively one could possibly argue that the variant allele mediates its effect on lung cancer risk by increasing the tendency to smoke more. During recent years several researchers have investigated or reviewed the role of nAChRs in lung cancer. ${ }^{26,44-48}$ Nicotine-derived nitrosamines are capable of activating $\mathrm{nAChRs}{ }^{49}$ promoting cell proliferation and apoptotic inhibition ${ }^{50}$, and both nicotine and 4-(methylnitrosamino)-1-(3-pyridyl)-1-butanon may stimulate Akt-dependent proliferation and NFkB-dependent reduction in apoptosis, ${ }^{51,52}$ providing plausible mechanisms for nicotine and its metabolites to promote disease development.

In conclusion, there is convincing evidence that the CHRNA5/ $A 3 / B 4$ gene cluster has an important role in both nicotine dependence, lung cancer and the loss of lung function. Our data suggest a role of rs16969968 in nicotine dependence rather than a direct effect on lung cancer risk and loss of lung function. However, as lung cancer is rare in never smokers, this hypothesis is difficult to test and a comprehensive meta-analysis will be required to obtain a sufficient sample size. To uncover the role of the CHRNA5/A3/B4 gene cluster the genetic variation in this cluster needs to be investigated in more detail possibly by sequencing in order to identify novel variants. To elucidate the role in lung carcinogenesis more functional studies of variant receptors need to be conducted.

\section{CONFLICT OF INTEREST}

The authors declare no conflict of interest.

\section{ACKNOWLEDGEMENTS}

'The study has used data from the Cancer Registry of Norway. The interpretation and reporting of these data are the sole responsibility of the authors, and no endorsement by the Cancer Registry of Norway is intended nor should be inferred.' The Nord-Trøndelag Health Study (The HUNT Study) is a collaboration between HUNT Research Centre (Faculty of Medicine, Norwegian University of Science and Technology NTNU), Nord-Trøndelag County Council, Central Norway Health Authority and the Norwegian Institute of Public Health. The lung study in HUNT 2 and 3 received nondemanding funding from AstraZeneca, Norway. This work was supported by The Norwegian Cancer Society, The Cancer Fund at St Olavs Hospital and Svanhild and Arne Must Fund for Medical Research.

1 World Health Organization. WHO Report on the Global Tobacco Epidemic, 2008 The MPOWER package: Geneva, Switzerland.

2 Wasswa-Kintu S, Gan WQ, Man SFP, Pare PD, Sin DD: Relationship between reduced forced expiratory volume in one second and the risk of lung cancer: a systematic review and meta-analysis. Thorax 2005; 60: 570-575.

3 Global Initiative for Chronic Obstructive Lung Disease: Global strategy for the diagnosis, management, and prevention of COPD: updated 2010. http://www.goldcopd org/uploads/users/files/GOLDReport_April112011.pdf.

4 Lopez AD, Mathers CD: Measuring the global burden of disease and epidemiological transitions: 2002-2030. Ann Trop Med Parasitol 2006; 100: 481-499.

5 Amos Cl, Wu X, Broderick P et al: Genome-wide association scan of tag SNPs identifies a susceptibility locus for lung cancer at 15q25.1. Nat Genet 2008; 40: 616-622.

6 Thorgeirsson TE, Geller F, Sulem P et al: A variant associated with nicotine dependence, lung cancer and peripheral arterial disease. Nature 2008; 452: 638-642.

7 Wang Y, Broderick P, Webb E et al: Common 5p15.33 and 6p21.33 variants influence lung cancer risk. Nat Genet 2008; 40: 1407-1409.

8 Broderick P, Wang Y, Vijayakrishnan J et al: Deciphering the impact of common genetic variation on lung cancer risk: a genome-wide association study. Cancer Res 2009; 69: 6633-6641.

9 Hung RJ, McKay JD, Gaborieau V et al: A susceptibility locus for lung cancer maps to nicotinic acetylcholine receptor subunit genes on 15q25. Nature 2008; 452: 633-637.
10 Lips EH, Gaborieau V, McKay JD et al: Association between a 15q25 gene variant, smoking quantity and tobacco-related cancers among 17000 individuals. Int J Epidemiol 2010; 39: 563-577.

11 Spitz MR, Amos CI, Dong Q, Lin J, Wu X: The CHRNA5-A3 region on chromosome $15 q 24-25.1$ is a risk factor both for nicotine dependence and for lung cancer. J Natl Cancer Inst 2008; 100: 1552-1556.

12 Truong T, Hung RJ, Amos $\mathrm{Cl}$ et al: Replication of lung cancer susceptibility loci at chromosomes 15q25, 5p15, and 6p21: a pooled analysis from the International Lung Cancer Consortium. J Natl Cancer Inst 2010; 102: 959-971.

13 Pillai SG, Ge D, Zhu G et al: A genome-wide association study in chronic obstructive pulmonary disease (COPD): identification of two major susceptibility loci. PLoS Genet 2009; 5: e1000421.

14 Bierut LJ, Madden PA, Breslau N et al: Novel genes identified in a high-density genome wide association study for nicotine dependence. Hum Mol Genet 2007; 16 24-35

15 Bierut LJ, Stitzel JA, Wang JC et al: Variants in nicotinic receptors and risk for nicotine dependence. Am J Psychiatry 2008; 165: 1163-1171.

16 Saccone NL, Saccone SF, Hinrichs AL et al: Multiple distinct risk loci for nicotine dependence identified by dense coverage of the complete family of nicotinic recepto subunit (CHRN) genes. Am J Med Genet B Neuropsychiatr Genet 2009; 150B: 453-466.

17 Saccone NL, Schwantes-An TH, Wang JC et al: Multiple cholinergic nicotinic receptor genes affect nicotine dependence risk in African and European Americans. Genes Brain Behav 2010; 9: 741-750.

18 Saccone NL, Wang JC, Breslau N et al: The CHRNA5-CHRNA3 CHRNB4 nicotinic receptor subunit gene cluster affects risk for nicotine dependence in African-Americans and in European-Americans. Cancer Res 2009; 69: 6848-6856.

19 Saccone SF, Hinrichs AL, Saccone NL et al: Cholinergic nicotinic receptor genes implicated in a nicotine dependence association study targeting 348 candidate genes with 3713 SNPs. Hum Mol Genet 2007; 16: 36-49.

20 Weiss RB, Baker TB, Cannon DS et al: A candidate gene approach identifies the CHRNA5-A3-B4 region as a risk factor for age-dependent nicotine addiction. PLoS Genet 2008; 4: e1000125.

21 Ware JJ, van den Bree MB, Munafo MR: Association of the CHRNA5-A3-B4 gene cluster with heaviness of smoking: a meta-analysis. Nicotine Tob Res 2011; 13 1167-1175.

22 Tobacco and Genetics Consortium; Furberg HKY, Dackor J, Boerwinkle E et al: Genome-wide meta-analyses identify multiple loci associated with smoking behavior Nat Genet 2010; 42: 441-447.

23 Liu JZ, Tozzi F, Waterworth DM et al: Meta-analysis and imputation refines the association of 15q25 with smoking quantity. Nat Genet 2010; 42: 436-440.

24 Thorgeirsson TE, Gudbjartsson DF, Surakka I et al: Sequence variants at CHRNB3-CHRNA6 and CYP2A6 affect smoking behavior. Nat Genet 2010; 42: 448-453.

25 Saccone NL, Culverhouse RC, Schwantes-An TH et al: Multiple independent loci at chromosome 15q25.1 affect smoking quantity: a meta-analysis and comparison with lung cancer and COPD. PLoS Genet 2010; 6: e1001053.

26 Improgo MR, Scofield MD, Tapper AR, Gardner PD: From smoking to lung cancer: the CHRNA5/A3/B4 connection. Oncogene 2010; 29: 4874-4884.

27 Dasgupta P, Chellappan SP: Nicotine-mediated cell proliferation and angiogenesis: new twists to an old story. Cell Cycle 2006; 5: 2324-2328.

28 Maneckjee R, Minna JD: Opioids induce while nicotine suppresses apoptosis in human lung cancer cells. Cell Growth Differ 1994; 5: 1033-1040.

29 Wright SC, Zhong J, Zheng H, Larrick JW: Nicotine inhibition of apoptosis suggests role in tumor promotion. FASEB J 1993; 7: 1045-1051.

30 Foulds J, Ramstrom L, Burke M, Fagerstrom K: Effect of smokeless tobacco (snus) on smoking and public health in Sweden. Tob Control 2003; 12: 349-359.

31 Holmen J, Midthjell K, Krüger $\varnothing$ et al: The Nord-Trøndelag Health Study 1995-97 (HUNT 2): objectives, contents, methods and participation. Norsk Epidemiol 2003, 13: 19-22.

32 Krokstad S, Langhammer A, Hveem K et al: Cohort Profile: The HUNT Study, Norway. Int J Epidemiol 2012; e-pub ahead of print 9 August 2012; doi:10.1093/ije/dys095.

33 Langhammer A, Johnsen R, Gulsvik A, Holmen TL, Bjermer L: Sex differences in lung vulnerability to tobacco smoking. Eur Respir J 2003; 21: 1017-1023.

34 Rostad H, Naalsund A, Norstein J, Jacobsen R, Aalokken TM: Is the treatment of lung cancer in Norway adequate? Tidsskr Nor Laegeforen 2002; 122: 2258-2262.

35 Langhammer A, Johnsen R, Gulsvik A, Holmen TL, Bjermer L: Forced spirometry reference values for Norwegian adults: the Bronchial Obstruction in Nord-Trondelag Study. Eur Respir J 2001; 18: 770-779.

36 Young RP, Hopkins RJ, Hay BA, Epton MJ, Black PN, Gamble GD: Lung cancer gene associated with COPD: triple whammy or possible confounding effect? Eur Respir J 2008; 32: 1158-1164.

37 Thorgeirsson TE, Stefansson K: Commentary: gene-environment interactions and smoking-related cancers. Int J Epidemiol 2010; 39: 577-579.

38 Lund KE, Scheffels J, McNeill A: The association between use of snus and quit rates for smoking: results from seven Norwegian cross-sectional studies. Addiction 2011 106: 162-167.

39 Piper ME, McCarthy DE, Baker TB: Assessing tobacco dependence: a guide to measure evaluation and selection. Nicotine Tobacco Res 2006; 8: 339-351.

40 Baker TB, Weiss RB, Bolt D et al: Human neuronal acetylcholine receptor A5-A3-B4 haplotypes are associated with multiple nicotine dependence phenotypes. Nicotine Tob Res 2009; 11: 785-796. 
41 Berrettini W, Yuan X, Tozzi F et al: Alpha-5/alpha-3 nicotinic receptor subunit alleles increase risk for heavy smoking. Mol Psychiatry 2008; 13: 368-373.

42 Hansen HM, Xiao Y, Rice T et al: Fine mapping of chromosome 15q25.1 lung cancer susceptibility in African-Americans. Hum Mol Genet 2010; 19: 3652-3661.

43 Wang Y, Broderick P, Matakidou A, Eisen T, Houlston RS: Chromosome 15q25 (CHRNA3-CHRNA5) variation impacts indirectly on lung cancer risk. PLoS One 2011; 6: e19085.

44 Catassi A, Servent D, Paleari L, Cesario A, Russo P: Multiple roles of nicotine on cell proliferation and inhibition of apoptosis: implications on lung carcinogenesis. Mutat Res 2008; 659: 221-231.

45 Egleton RD, Brown KC, Dasgupta P: Nicotinic acetylcholine receptors in cancer: multiple roles in proliferation and inhibition of apoptosis. Trends Pharmacol Sci 2008; 29: 151-158.

46 Improgo MR, Scofield MD, Tapper AR, Gardner PD: The nicotinic acetylcholine receptor CHRNA5/A3/B4 gene cluster: dual role in nicotine addiction and lung cancer. Prog Neurobiol 2010; 92: 212-226.

47 Improgo MR, Schlichting NA, Cortes RY, Zhao-Shea R, Tapper AR, Gardner PD: ASCL1 regulates the expression of the CHRNA5/A3/B4 lung cancer susceptibility locus. Mol Cancer Res 2010; 8: 194-203.

48 Improgo MR, Tapper AR, Gardner PD: Nicotinic acetylcholine receptor-mediated mechanisms in lung cancer. Biochem Pharmacol 2011; 82: 1015-1021.

49 Schuller HM, Orloff M: Tobacco-specific carcinogenic nitrosamines. Ligands for nicotinic acetylcholine receptors in human lung cancer cells. Biochem Pharmacol 1998; 55: 1377-1384.

50 Schuller HM: Is cancer triggered by altered signalling of nicotinic acetylcholine receptors? Nat Rev Cancer 2009; 9: 195-205.

51 Tsurutani J, Castillo SS, Brognard J et al: Tobacco components stimulate Akt-dependent proliferation and NFkappaB-dependent survival in lung cancer cells. Carcinogenesis 2005; 26: 1182-1195.

52 West KA, Brognard J, Clark AS et al: Rapid Akt activation by nicotine and a tobacco carcinogen modulates the phenotype of normal human airway epithelial cells. J Clin Invest 2003; 111: 81-90.

(c)

SOMERIHHSTESER

mons Attribution-NonCommercial-No Derivative

Works 3.0 Unported License. To view a copy of this license, visit http://creativecommons.org/licenses/by-nc-nd/3.0/ 